\title{
The effect of transcranial direct current stimulation: a role for cortical excitation/inhibition balance?
}

\author{
Beatrix Krause $^{1}{ }^{*}$, Javier Márquez-Ruiz ${ }^{2}$ and Roi Cohen Kadosh ${ }^{1}$ \\ ${ }^{1}$ Department of Experimental Psychology, University of Oxford, Oxford, Oxfordshire, UK \\ ${ }^{2}$ Division of Neurosciences, Pablo de Olavide University, Seville, Spain
}

\section{Edited by:}

Marom Bikson, The City College of New York of The City University of New York, USA

Reviewed by:

Flavio Frohlich, University of North

Carolina - Chapel Hill, USA

Davide Reato, The City College of The

City University of New York, USA

\section{*Correspondence:}

Beatrix Krause, Department of

Experimental Psychology, University

of Oxford, 9 South Parks Road,

Oxford, Oxfordshire, OXI $3 \cup D, U K$

e-mail: beatrix.krause@psy.ox.ac.uk
Transcranial direct current stimulation (tDCS) is a promising tool for cognitive enhancement and neurorehabilitation in clinical disorders in both cognitive and clinical domains (e.g., chronic pain, tinnitus). Here we suggest the potential role of tDCS in modulating cortical excitation/inhibition (E/I) balance and thereby inducing improvements. We suggest that part of the mechanism of action of tDCS can be explained by non-invasive modulations of the E/l balance.

Keywords: transcranial direct current stimulation (tDCS), excitation, inhibition, GABA, glutamate, cognition

\section{INTRODUCTION}

Cognitive enhancement is a popular topic in the neuroscience community. Non-invasive neuromodulation methods, such as transcranial direct current stimulation (tDCS) can either increase (e.g., anodal) or decrease (e.g., cathodal) cortical excitability (Nitsche and Paulus, 2001; Nitsche et al., 2003) and thereby modulate cortical activity levels.

At a cellular level, the applied external electric field modifies the transmembrane potential differences by forcing the displacement of intracellular ions which cancel the generated intracellular field and thereby modify the spike firing probability (Bikson et al., 2004; Ruffini et al., 2013). With sufficient tDCS duration, synaptically driven aftereffects are induced (Bindman et al., 1964). The final effects of tDCS depend on the individual neural morphology (Radman et al., 2009), the orientation of somato-dendritic axes, and the neural pathways with respect to the electric field (Bikson et al., 2004; Kabakov et al., 2012).

tDCS has positive effects in a variety of clinical conditions such as Parkinson's disease, tinnitus, chronic pain, stroke, and even childhood psychosis (e.g., Fregni et al., 2006; Song et al., 2012; David et al., 2013; Khedr et al., 2013; Moreno-Duarte et al., 2013), but also in healthy individuals (Jacobson et al., 2012; Kuo and Nitsche, 2012; Cohen Kadosh, 2013). It is therefore considered a promising neurorehabilitation tool. Moreover, tDCS has recently been suggested as a possible tool to improve learning disabilities in children (Krause and Cohen Kadosh, 2013; Vicario and Nitsche, 2013). A crucial question remains to be answered: how exactly does tDCS modify such diverse conditions in both the typical and atypical brain?

\section{NEUROTRANSMITTERS AND tDCS}

Magnetic resonance spectroscopy (MRS) studies have shown that anodal tDCS reduces local concentrations of the inhibitory neurotransmitter gamma-aminobutyric acid (GABA), whereas cathodal tDCS reduces excitatory glutamate levels (Stagg et al., 2009; Clark et al., 2011).

Others have suggested that local GABA reductions co-occur with learning and performance improvements (Floyer-Lea et al., 2006) and that the magnitude of regional GABAergic changes during anodal tDCS reflects the degree of learning (Stagg et al., 2011). Namely, the further GABA is decreased, the larger the observed learning effect. Such disinhibition may lead to the unmasking of hidden excitatory connections (Jacobs and Donoghue, 1991) and thereby allow for the induction of activity-dependent long-term potentiation (LTP). LTP in turn is capable of inducing cortical reorganization, most likely by increasing local synaptic effectiveness (Hess and Donoghue, 1994), which in turn might alter deficient network processing.

In addition, data coming from animal experiments have demonstrated the implication of N-methyl-D-aspartate (NMDA) receptors and brain-derived neurotrophic factor (BDNF) in the synaptic potentiation of the motor cortex after anodal tDCS (Fritsch et al., 2010). Moreover, local administration of the adenosine A1 receptor antagonist 8-cyclopentyl-1,3dipropylxanthine (DCPCX) in the somatosensory cortex of alert rabbits prevented long-term depression induced by cathodal tDCS (Marquez-Ruiz et al., 2012). These data suggest that beyond GABA and glutamate, other neurochemicals may be involved in the mechanisms underlying long-term tDCS effects.

\section{EXCITATION/INHIBITION (E/I) BALANCE}

Homeostatic control of cortical excitability and induction of plasticity are crucial for allowing efficient information transfer in the brain, (Turrigiano and Nelson, 2000). This means that while plastic changes occur, the network must still maintain a certain amount of stability in order to produce meaningful 
output. The dysregulation of cortical excitability may thus lead to symptoms seen in various central nervous system disorders (Eichler and Meier, 2008), depending on the area(s) in which the imbalance occurs. For instance, regional abnormalities in GABA concentrations have been found in neuropsychiatric disorders, such as schizophrenia (Goto et al., 2009; Yoon et al., 2010; Yizhar et al., 2011; Rowland et al., 2013), autism (Kubas et al., 2012; Rojas et al., 2013), insomnia (Morgan et al., 2012), and panic disorder (Long et al., 2013).

However, GABA concentrations alone may not fully explain different kinds of cognitive deficits. For instance, if glutamatergic excitation is increased as well, we would not expect to observe performance abnormalities. Most studies so far have only looked at glutamate and GABA in isolation (e.g., Goto et al., 2009; Yoon et al., 2010; Kubas et al., 2012; Rojas et al., 2013).

We suggest that the regional cortical excitation/inhibition (E/I) balance, measured by ratios of glutamate/GABA, may provide more meaningful interpretations of individual cognitive performance and deficits than glutamate or GABA alone. GABA and glutamate contribute in a complementary fashion to high-level prefrontal cognitive performance in healthy adults (Jocham et al., 2012). Furthermore, individuals with autism or schizophrenia show higher E/I ratios compared to healthy controls (Rubenstein and Merzenich, 2003), and this has been suggested to be related to behavioral and cognitive deficits (Yizhar et al., 2011). Similarly, regional increases in glutamate (Carrey et al., 2007; Arcos-Burgos et al., 2012) and reduced levels of GABA (Edden et al., 2012) have been found in several different brain areas of individuals with Attention-deficit hyperactivity disorder (ADHD). These findings lend support to the view that E/I balance plays a major role in normal cognition, as well as the symptomatic patterns of a variety of clinical conditions.

Using cathodal tDCS to artificially decrease E/I in ADHD for example could be beneficial. Cathodal stimulation may restore the elevated E/I balance towards a more typical level in targeted regions, which require greater baseline inhibition, in order to reduce irrelevant output. For instance, in healthy adults, applying cathodal stimulation to prefrontal regions has been shown to lead to improved attentional processing. This likely enhances prefrontal filtering of irrelevant information (Weiss and Lavidor, 2012).

The direction of the E/I imbalance may determine the behavioral outcome depending on the particular brain area and appears to be different in different clinical populations. Therefore, a fundamental understanding of individual differences in E/I ratio would allow for optimization of the choice of tDCS parameters for each individual in terms of polarity, intensity, duration, etc. (Figure 1).

\section{DISCUSSION}

This simple, but elegant model explains individual differences in cognitive performance and cognitive deficits, as well as the polarity-specific effects of tDCS on cognition, and can be extended to non-cognitive domains, as well (e.g., pain: Harris and
A

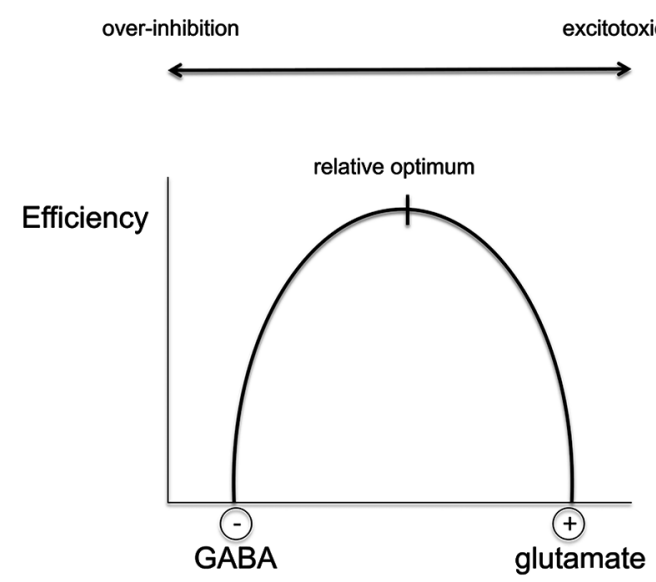

Activation

FIGURE 1 | The relationship between excitation/inhibition (E/I) balance and efficiency of a given cortical region. (A) According to the current hypothesis, E/l balance within a brain area can be viewed as an inverted-U shape in which the optimal performance is achieved when excitation and inhibition interact efficiently, allowing for both plasticity and stability. The degree of baseline $\mathrm{E} / \mathrm{l}$ might, however, differ per brain region and individual. If the optimal balance is achieved, homeostatic control of activity-dependent plasticity and synaptic efficiency are possible and can lead to meaningful behavioral output. Deviations from the ideal balance are associated with atypical behavior and the severity of the deficit may vary with the degree of imbalance. tDCS can be used to target and restore the individual abnormalities in $\mathrm{E} / \mathrm{I}$ imbalance in different neurological conditions. Only a
B

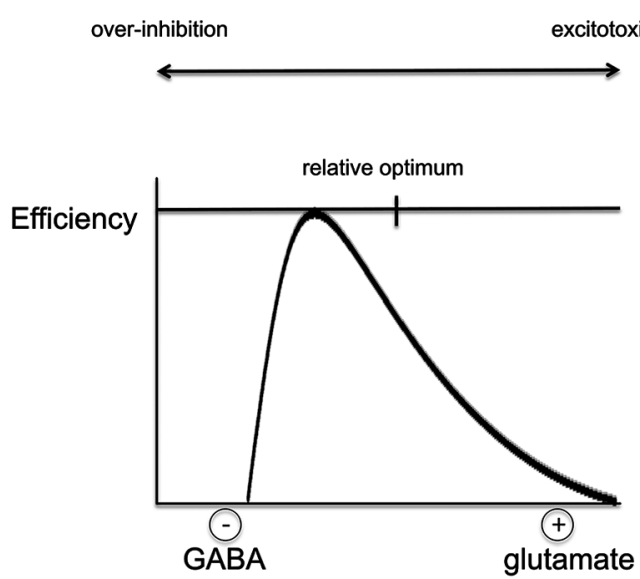

Activation

moderate level of activation, i.e., balanced E/l levels, can reach the optimal level of processing efficiency and allow for homeostatic plasticity. High levels of GABA can lead to cortical over-inhibition that will reduce network output, whereas hyperactive glutamatergic activity can lead to excessive output and eventually to excitotoxicity and cell death (Faden et al., 1989; Belousov, 2012). (B) An example of the distribution of $E / l$ balance in the healthy population: the finding that most anodal tDCS studies report behavioral improvements suggests that the distribution may be skewed with the majority showing non-optimal E/I ratio. However, for some individuals with increased E/I ratios, anodal tDCS will shift the non-pathological imbalance even further towards over-activation and therefore reduce behavioral outcomes. 
Clauw, 2012). Nevertheless, the effects of tDCS on neural network dynamics, more specifically at neurotransmitters concentrations, are largely unknown.

At a microscopic level, glutamate is released by pyramidal cell synapses and thalamic synaptic inputs, whereas GABA is mainly released by a variety of interneurons (Nicoll et al., 1990; McCormick, 1992). Animal experiments using brain slices suggest that pyramidal cells in layer $\mathrm{V}$ are the most sensitive to the effects of weak electric fields applied over the skull surface (Radman et al., 2009). Thus, anodal and cathodal tDCS are expected to increase or decrease, respectively, the membrane potential of pyramidal cells and thereby alter the glutamatergic tone in the cortex.

Nevertheless, glutamate levels not only depend on pyramidal cells but also on input from thalamic projections. It has been recently shown in both humans (Polania et al., 2012) and alert rabbits (Marquez-Ruiz et al., 2012) that tDCS also modifies thalamocortical synapses by means of glutamate release from sensory afferents. As pyramidal cells project to different types of interneurons, it is expected that the modulation of glutamate levels correlates with GABA release. However, a recent computational modeling study based on in-vivo experimental data proposed that tDCS may induce opposing effects on different types of interneurons (Molaee-Ardekani et al., 2013), suggesting a more complex scenario. Finally, in order to fully understand the mechanism underlying E/I balance, other factors, such as levels of BDNF or cortical adenosine and cortical oscillations

\section{REFERENCES}

Arcos-Burgos, M., Londono, A. C., Pineda, D. A., Lopera, F., Palacio, J. D., Arbelaez, A., et al. (2012). Analysis of brain metabolism by proton magnetic resonance spectroscopy (1H-MRS) in attentiondeficit/hyperactivity disorder suggests a generalized differential ontogenic pattern from controls. Atten. Defic. Hyperact. Disord. 4, 205-212. doi: 10.1007/s12402-012-0088-0

Atallah, B. V., and Scanziani, M. (2009). Instantaneous modulation of gamma oscillation frequency by balancing excitation with inhibition. Neuron 62, 566-577. doi: 10.1016/j. neuron.2009.04.027

Belousov, A. B. (2012). Novel model for the mechanisms of glutamatedependent excitotoxicity: role of neuronal gap junctions. Brain Res. 1487, 123-130. doi: 10.1016/j. brainres.2012.05.063

Bikson, M., Inoue, M., Akiyama, H., Deans, J. K., Fox, J. E., Miyakawa, H., et al. (2004). Effects of uniform extracellular DC electric fields on excitability in rat hippocampal slices in vitro. J. Physiol. 557, 175190. doi: 10.1113/jphysiol.2003. 055772

Bindman, L. J., Lippold, O. C., and Redfearn, J. W. (1964). The action cerebral cortex of the rat (1) during current flow and (2) in the production of long-lasting after-effects. J. Physiol. 172, 369-382.

Carrey, N. J., Macmaster, F. P., Gaudet, L., and Schmidt, M. H. (2007). Striatal creatine and glutamate/glutamine in attentiondeficit/hyperactivity disorder. J. Child Adolesc. Psychopharmacol. 17, 11-17. doi: 10.1089/cap.2006.0008

Clark, V. P., Coffman, B. A., Trumbo, M. C., and Gasparovic, C. (2011). Transcranial direct current stimulation ( $\mathrm{tDCS}$ ) produces localized and specific alterations in neurochemistry: a (1)H magnetic resonance spectroscopy study. Neurosci. Lett. 500, 67-71. doi: 10.1016/j.neulet. 2011.05.244

Cohen Kadosh, R. (2013). Using transcranial electrical stimulation to enhance cognitive functions in the typical and atypical brain. Transl. Neurosci. 4, 20-33. doi: 10. 2478/s13380-013-0104-7

David, C. N., Rapoport, J. L., and Gogtay, N. (2013). Treatments in context: transcranial direct current brain stimulation as a potential treatment in pediatric psychosis. Expert Rev. Neurother. 13, 447-458. doi: 10.1586/ern.13.29 of brief polarizing currents on the must also be taken into consideration. For example, it has been shown in brain slices that weak direct current (DC) stimulation may modulate slow-wave (Frohlich and McCormick, 2010) and gamma oscillations (Reato et al., 2010) related with E/I balance in the cortex (Shu et al., 2003; Haider et al., 2006; Atallah and Scanziani, 2009).

According to the current evidence, tDCS is likely to reinstate an optimal E/I balance that allows for optimal homeostatic plasticity in learning and cognition, if applied adequately to each individual's predispositions. If this consistently proves to be the case, a variety of cortex-based clinical conditions including atypical brain development may be successfully treated using tDCS. So far, there is little research investigating the relationship between E/I balance and cognition. The assessment of this balance in different clinical, neurological and neuro-developmental disorders will help refine tDCS strategies for treatment in the future. Whether electrical stimulation can also modulate E/I balance in the case of transcranial random noise stimulation (tRNS) and transcranial alternating current stimulation (tACS) is currently unknown and requires further exploration.

\section{ACKNOWLEDGMENTS}

We would like to thank Rebecca Merkley for her comments. Beatrix Krause is supported by the Economic and Social Research Council (ESRC) and the Studienstiftung des Deutschen Volkes. Roi Cohen Kadosh is supported by the Wellcome Trust (WT88378).
Edden, R. A., Crocetti, D., Zhu, H., Gilbert, D. L., and Mostofsky, S. H. (2012). Reduced GABA concentration in attention-deficit/ hyperactivity disorder. Arch. Gen. Psychiatry 69, 750-753. doi: 10. 1001/archgenpsychiatry.2011.2280

Eichler, S. A., and Meier, J. C. (2008). E-I balance and human diseases - from molecules to networking. Front. Mol. Neurosci. 1:2. doi: 10. 3389/neuro.02.002.2008

Faden, A. I., Demediuk, P., Panter, S. S., and Vink, R. (1989). The role of excitatory amino acids and NMDA receptors in traumatic brain injury. Science 244, 798-800. doi: 10. 1126/science. 2567056

Floyer-Lea, A., Wylezinska, M., Kincses, T., and Matthews, P. M. (2006). Rapid modulation of GABA concentration in human sensorimotor cortex during motor learning. J. Neurophysiol. 95, 1639-1644. doi: 10. 1152/jn.00346.2005

Fregni, F., Boggio, P. S., Santos, M. C., Lima, M., Vieira, A. L., Rigonatti, S. P., et al. (2006). Noninvasive cortical stimulation with transcranial direct current stimulation in Parkinson's disease. Mov. Disord. 21, 1693-1702. doi: 10.1002/mds. 21012

Fritsch, B., Reis, J., Martinowich, K., Schambra, H. M., Ji, Y., Cohen, L.
G., et al. (2010). Direct current stimulation promotes BDNF-dependent synaptic plasticity: potential implications for motor learning. Neuron 66, 198-204. doi: 10.1016/j.neuron. 2010.03.035

Frohlich, F., and McCormick, D. A. (2010). Endogenous electric fields may guide neocortical network activity. Neuron 67, 129-143. doi: 10.1016/j.neuron.2010.06.005

Goto, N., Yoshimura, R., Moriya, J., Kakeda, S., Ueda, N., IkenouchiSugita, A., et al. (2009). Reduction of brain gamma-aminobutyric acid (GABA) concentrations in earlystage schizophrenia patients: 3T Proton MRS study. Schizophr. Res. 112, 192-193. doi: 10.1016/j.schres. 2009.04.026

Haider, B., Duque, A., Hasenstaub, A R., and McCormick, D. A. (2006). Neocortical network activity in vivo is generated through a dynamic balance of excitation and inhibition. J. Neurosci. 26, 4535-4545. doi: 10. 1523/jneurosci.5297-05.2006

Harris, R. E., and Clauw, D. J. (2012). Imaging central neurochemical alterations in chronic pain with proton magnetic resonance spectroscopy. Neurosci. Lett. 520, 192-196. doi: 10.1016/j.neulet.2012. 03.042 
Hess, G., and Donoghue, J. P. (1994). Long-term potentiation of horizontal connections provides a mechanism to reorganize cortical motor maps. J. Neurophysiol. 71, 25432547.

Jacobs, K. M., and Donoghue, J. P. (1991). Reshaping the cortical motor map by unmasking latent intracortical connections. Science 251, 944-947. doi: 10.2307/2874910

Jacobson, L., Koslowsky, M., and Lavidor, M. (2012). tDCS polarity effects in motor and cognitive domains: a meta-analytical review. Exp. Brain Res. 216, 1-10. doi: 10.1007/s00221011-2891-9

Jocham, G., Hunt, L. T., Near, J., and Behrens, T. E. (2012). A mechanism for value-guided choice based on the excitation-inhibition balance in prefrontal cortex. Nat. Neurosci. 15, 960-961. doi: 10.1038/nn.3140

Kabakov, A. Y., Muller, P. A., PascualLeone, A., Jensen, F. E., and Rotenberg, A. (2012). Contribution of axonal orientation to pathwaydependent modulation of excitatory transmission by direct current stimulation in isolated rat hippocampus. J. Neurophysiol. 107, 1881-1889. doi: $10.1152 /$ jn.00715.2011

Khedr, E. M., Shawky, O. A., ElHammady, D. H., Rothwell, J. C., Darwish, E. S., Mostafa, O. M., et al. (2013). Effect of anodal versus cathodal transcranial direct current stimulation on stroke rehabilitation: a pilot randomized controlled trial. Neurorehabil. Neural Repair 27, 592-601. doi: 10. 1177/1545968313484808

Krause, B., and Cohen Kadosh, R. (2013). Can transcranial electrical stimulation improve learning difficulties in atypical brain development? A future possibility for cognitive training. Dev. Cogn. Neurosci. doi: 10.1016/j.den.2013.04. 001. [Epub ahead of print].

Kubas, B., Kulak, W., Sobaniec, W., Tarasow, E., Lebkowska, U., and Walecki, J. (2012). Metabolite alterations in autistic children: a $1 \mathrm{H} \mathrm{MR}$ spectroscopy study. Adv. Med. Sci. 57, 152-156. doi: 10.2478/v10039012-0014-x

Kuo, M.-F., and Nitsche, M. A. (2012). Effects of transcranial electrical stimulation on cognition. Clin. EEG Neurosci. 43, 192-199. doi: 10. 1177/1550059412444975

Long, Z., Medlock, C., Dzemidzic, M., Shin, Y. W., Goddard, A. W., and Dydak, U. (2013). Decreased GABA levels in anterior cingulate cortex/medial prefrontal cortex in panic disorder. Prog. Neu- ropsychopharmacol. Biol. Psychiatry 44, 131-135. doi: 10.1016/j.pnpbp. 2013.01.020

Marquez-Ruiz, J., Leal-Campanario, R., Sanchez-Campusano, R., MolaeeArdekani, B., Wendling, F., Miranda, P. C., et al. (2012). Transcranial direct-current stimulation modulates synaptic mechanisms involved in associative learning in behaving rabbits. Proc. Natl. Acad. Sci. U S A 109, 6710-6715. doi: 10.1073/pnas. 1121147109

McCormick, D. A. (1992). Neurotransmitter actions in the thalamus and cerebral cortex and their role in neuromodulation of thalamocortical activity. Prog. Neurobiol. 39, 337-388. doi: 10.1016/03010082(92)90012-4

Molaee-Ardekani, B., Marquez-Ruiz, J., Merlet, I., Leal-Campanario, R., Gruart, A., Sanchez-Campusano, R., et al. (2013). Effects of transcranial Direct Current Stimulation (tDCS) on cortical activity: a computational modeling study. Brain Stimul. 6, 25-39. doi: 10.1016/j.brs.2011. 12.006

Moreno-Duarte, I., Morse, L., Alam, M., Bikson, M., Zafonte, R., and Fregni, F. (2013). Targeted therapies using electrical and magnetic neural stimulation for the treatment of chronic pain in spinal cord injury. Neuroimage doi: 10.1016/j. neuroimage.2013.05.097. [Epub ahead of print].

Morgan, P. T., Pace-Schott, E. F., Mason, G. F., Forselius, E., Fasula, M., Valentine, G. W., et al. (2012). Cortical GABA levels in primary insomnia. Sleep 35, 807-814. doi: $10.5665 /$ sleep. 1880

Nicoll, R. A., Malenka, R. C., and Kauer, J. A. (1990). Functional comparison of neurotransmitter receptor subtypes in mammalian central nervous system. Physiol. Rev. 70, 513-565.

Nitsche, M. A., and Paulus, W. (2001). Sustained excitability elevations induced by transcranial DC motor cortex stimulation in humans. Neurology 57, 1899-1901. doi: 10. 1212/wnl.57.10.1899

Nitsche, M. A., Nitsche, M. S., Klein, C. C., Tergau, F., Rothwell, J. C., and Paulus, W. (2003). Level of action of cathodal DC polarisation induced inhibition of the human motor cortex. Clin. Neurophysiol. 114, 600-604. doi: 10.1016/s13882457(02)00412-1

Polania, R., Paulus, W., and Nitsche, M. A. (2012). Modulating corticostriatal and thalamo-cortical functional connectivity with transcranial direct current stimulation. Hum. Brain Mapp. 33, 2499-2508. doi: 10. 1002/hbm. 21380

Radman, T., Ramos, R. L., Brumberg, J. C., and Bikson, M. (2009). Role of cortical cell type and morphology in subthreshold and suprathreshold uniform electric field stimulation in vitro. Brain Stimul. 2, 215228, 228.e1-3. doi: 10.1016/j.brs. 2009.03.007

Reato, D., Rahman, A., Bikson, M., and Parra, L. C. (2010). Low-Intensity electrical stimulation affects network dynamics by modulating population rate and spike timing. $J$. Neurosci. 30, 15067-15079. doi: 10. 1523/jneurosci.2059-10.2010

Rojas, D. C., Singel, D., Steinmetz, S., Hepburn, S., and Brown, M. S. (2013). Decreased left perisylvian GABA concentration in children with autism and unaffected siblings. Neuroimage doi: 10.1016/j. neuroimage.2013.01.045. [Epub ahead of print].

Rowland, L. M., Kontson, K., West, J., Edden, R. A., Zhu, H., Wijtenburg, S. A., et al. (2013). In vivo measurements of Glutamate, GABA, and NAAG in Schizophrenia. Schizophr. Bull. 39, 1096-1104. doi: 10.1093/schbul/sbs092

Rubenstein, J. L., and Merzenich, M. M. (2003). Model of autism: increased ratio of excitation/inhibition in key neural systems. Genes Brain Behav. 2, 255-267.

Ruffini, G., Wendling, F., Merlet, I., Molaee-Ardekani, B., Mekonnen, A., Salvador, R., et al. (2013). Transcranial current brain stimulation (tCS): models and technologies. IEEE Trans. Neural Syst. Rehabil. Eng. 21, 333-345. doi: 10. 1109/tnsre.2012.2200046

Shu, Y., Hasenstaub, A., and McCormick, D. A. (2003). Turning on and off recurrent balanced cortical activity. Nature 423, 288-293. doi: 10.1038/nature01616

Song, J. J., Vanneste, S., Van De Heyning, P., and De Ridder, D. (2012). Transcranial direct current stimulation in tinnitus patients: a systemic review and meta-analysis. ScientificWorldJournal 2012:427941. doi: 10. 1100/2012/427941

Stagg, C. J., Bachtiar, V., and JohansenBerg, H. (2011). The role of GABA in human motor learning. Curr. Biol. 21, 480-484. doi: 10.1016/j. cub.2011.01.069

Stagg, C. J., Best, J. G., Stephenson, M. C., O'shea, J., Wylezinska, M., Kincses, Z. T., et al. (2009). Polaritysensitive modulation of cortical neurotransmitters by transcranial stimulation. J. Neurosci. 29, 52025206. doi: 10.1523/JNEUROSCI. 4432-08.2009

Turrigiano, G. G., and Nelson, S. B. (2000). Hebb and homeostasis in neuronal plasticity. Curr. Opin. Neurobiol. 10, 358-364. doi: 10. 1016/s0959-4388(00)00091-x

Vicario, C. M., and Nitsche, M. A. (2013). Transcranial direct current stimulation: a remediation tool for the treatment of childhood congenital dyslexia? Front. Hum. Neurosci. 7:139. doi: 10.3389/fnhum. 2013.00139

Weiss, M., and Lavidor, M. (2012). When less is more: evidence for a facilitative cathodal tDCS effect in attentional abilities. J. Cogn. Neurosci. 24, 1826-1833. doi: 10. 1162/jocn_a_00248

Yizhar, O., Fenno, L. E., Prigge, M., Schneider, F., Davidson, T. J., O'shea, D. J., et al. (2011). Neocortical excitation/inhibition balance in information processing and social dysfunction. Nature 477, 171-178. doi: 10.1038/nature10360

Yoon, J. H., Maddock, R. J., Rokem, A., Silver, M. A., Minzenberg, M. J., Ragland, J. D., et al. (2010). GABA concentration is reduced in visual cortex in schizophrenia and correlates with orientation-specific surround suppression. J. Neurosci. 30, 3777-3781. doi: 10.1523/jneurosci. 6158-09.2010

Conflict of interest: Roi Cohen Kadosh filed a patent for an apparatus for improving and/or maintaining numerical ability.

Received: 14 June 2013; accepted: 04 September 2013; published online: 24 September 2013.

Citation: Krause B, Márquez-Ruiz J and Cohen Kadosh $R$ (2013) The effect of transcranial direct current stimulation: a role for cortical excitation/inhibition balance? Front. Hum. Neurosci. 7:602. doi 10.3389/fnhum.2013.00602

This article was submitted to the journal Frontiers in Human Neuroscience. Copyright $\odot 2013$ Krause, Márquez-Ruiz and Cohen Kadosh. This is an openaccess article distributed under the terms of the Creative Commons Attribution License (CC BY). The use, distribution or reproduction in other forums is permitted, provided the original author (s) or licensor are credited and that the original publication in this journal is cited, in accordance with accepted academic practice. No use, distribution or reproduction is permitted which does not comply with these terms. 